\title{
Severity of degenerative lumbar spinal stenosis affects pelvic rigidity during walking
}

\author{
Helen Bumann ${ }^{1}$ \\ Corina Nüesch $2,3,4$ \\ Stefan Loske ${ }^{2}$ \\ S. Kimberly Byrnes 2,5 \\ Balázs Kovacs ${ }^{6}$ \\ Ruben Janssen ${ }^{6}$ \\ Stefan Schären ${ }^{1}$ \\ Annegret Mündermann ${ }^{2,3,4}$ \\ Cordula Netzer ${ }^{1}$
${ }^{1}$ Department of Spinal Surgery, University Hospital Basel, Basel, Switzerland
${ }^{2}$ Department of Orthopaedics and Traumatology, University Hospital Basel, Basel, Switzerland
${ }^{3}$ Department of Biomedical Engineering, University of Basel, Basel, Switzerland
${ }^{4}$ Department of Clinical Research, University of Basel, Basel, Switzerland
${ }^{5}$ Faculty for Sport and Health Science, Technische Universität München, Munich, Germany
${ }^{6}$ Department of Radiology University Hospital Basel, Basel, Switzerland

\section{Clinical Observational Study}

August 2019

Submitted to: The Spine Journal

Manuscript \#SPINEE-D-19-00348

Address of correspondence:

\author{
Dr. med. Cordula Netzer \\ Department of Spinal Surgery \\ University Hospital Basel \\ Spitalstrasse 21 \\ 4031 Basel, Switzerland \\ Tel. +49613287675 \\ Email cordula.netzer@usb.ch
}

Word Count: 3948 words

Conflicts of Interest and Source of Funding: The authors declare no conflict of interest. This study was funded by the Departments of Spinal Surgery and Orthopaedics and Traumatology of the University Hospital Basel, Switzerland.

Acknowledgements: The authors thank Arik Rehani Musagara for his assistance in processing the MR image data. 


\begin{abstract}
Background Context. To understand the role of compensation mechanisms in the development and treatment of symptomatic degenerative lumbar spinal stenosis (DLSS), pelvic stability during walking should be objectively assessed in the context of clinical parameters.

Purpose. To determine the association among duration of symptoms, lumbar muscle atrophy, disease severity, pelvic stability during walking and surgical outcome in patients with DLSS scheduled for decompression surgery.

Study Design/Setting. Prospective observational study with intervention.

Patient Sample. Patients with symptomatic DLSS.
\end{abstract}

Outcome Measures. Oswestry Disability Index (ODI) score; duration of symptoms; lumbar muscle atrophy; severity grade; pelvis rigidity during walking.

Methods. Patients with symptomatic DLSS were analyzed on the day before surgery and 10 weeks and 12 months postoperatively. Duration of symptoms were categorized as: <2years, $<5$ years, and $>5 y e a r s$. Muscle atrophy at the stenosis level was categorized according to Goutallier. Bilateral cross-sectional areas (CSA) of the erector spinae and psoas muscles were quantified from MRIs. Stenosis grade was assessed using the Schizas classification. Pelvic tilt was measured in standing radiographs. Pelvic rigidity during walking was assessed as root mean square of the pelvic acceleration in each direction (antero-posterior, medio-lateral, vertical) normalized to walking speed measured using an inertial sensor attached to the skin between the posterior superior iliac spine.

Results. Body mass index but not duration of symptoms, lumbar muscle atrophy, pelvic rigidity and stenosis grade explained changes in ODI from before to after surgery. Patients with greater stenosis grade had greater pelvic rigidity during walking. Lumbar muscle atrophy did not 
correlate with pelvic rigidity during walking. Patients with lower stenosis grade had greater muscle atrophy and patients with smaller erector spinae and psoas muscle CSA had a greater pelvis tilt.

Conclusions. Greater pelvic rigidity during walking may represent a compensatory mechanism of adopting a protective body position to keep the spinal canal more open during walking and hence reduce pain. Pelvic rigidity during walking may be a useful screening parameter for identifying early compensating mechanisms. Whether it can be used as a parameter for personalized treatment planning or outcome prognosis necessitates further evaluation.

Level of evidence. Level 2, cohort study

Key Words: lumbar spinal stenosis; decompression surgery; pelvic rigidity; inertial sensor gait analysis 


\section{Introduction}

Degenerative lumbar spinal stenosis (DLSS) is one of the most frequent conditions of the spine and indication for spinal surgery in patients aged over 65 years [1]. The prevalence of DLSS is estimated at $7.3 \%$ in the adult population [2]. DLSS is characterized by diminished space for neural and vascular elements of the lumbar spinal canal in association with buttock or lower extremity pain and possibly neurological radicular deficits, which may occur with or without back pain. Often, the symptoms are aggravated in standing and walking position [3].

Previous studies have reported greater paraspinal muscle atrophy and fatty infiltration in patients with DLSS than in control groups $[4,5]$. Moreover, greater changes in muscle atrophy have been associated with lower function (higher Oswestry Disability Index (ODI) and pain interference scores) [6]. Nonetheless, among patients with DLSS, muscle atrophy or fatty infiltration do not correlate with the degree of stenosis [6]. Generally, the duration of symptoms vary largely among patients with DLSS and might play an important role in the development of muscular atrophy [7]. However, to date, evidence for a possible association of duration of symptoms and muscular atrophy is lacking.

Worse symptoms in standing and walking positions may be biomechanically explained by the additional narrowing of the spinal canal caused by increased lumbar lordosis in these positions compared to sitting positions [8]. Patients with DLSS not only have greater radiographically measured pelvis tilt and smaller lumbar flexion measured from radiographs than healthy persons $[1,9]$, those with greater pelvic tilt also had greater pain [10]. In contrast, during walking patients with DLSS have a smaller range of pelvic tilt and smaller range of lumbar flexion than 
healthy persons [9] which may be described as greater pelvic rigidity during walking in patients with DLSS. Moreover, differences in gait quality (spatiotemporal parameters and gait asymmetry) in patients with symptomatic DLSS compared to healthy subjects have been reported [11-14]. These studies imply that pelvic rigidity plays an important role in DLSS and its aggravated symptoms in upright positions.

The objective of this study was to determine if an association exists among duration of symptoms, lumbar muscle atrophy, degree of stenosis and pelvic rigidity during walking in patients with symptomatic DLSS treated with decompression surgery. The term "pelvic rigidity" is defined as smaller pelvic accelerations during walking. We hypothesized that (i) duration of symptoms, lumbar muscle atrophy, pelvic rigidity and degree of stenosis predict clinical outcome of decompression surgery and (ii) lumbar muscle atrophy and degree of stenosis correlate with pelvic rigidity during walking and standing. We further expected that pelvic rigidity during walking in patients with DLSS differ from that in age-matched asymptomatic healthy persons and changes after decompression surgery.

\section{Patients and methods}

Study cohort: An existing database from a previous observational study carried out at our clinic between June and September 2016 was used [14]. Patients with symptomatic DLSS, who had already been diagnosed and scheduled for surgery in our outpatient clinic were recruited. The study was approved by the regional ethics committee, and all included patients signed the informed consent form. Inclusion criteria were: age $>40$ years; clinical symptoms for at least 6 months; intermittent neurogenic claudication with limitations of their walking ability in one or 
both legs; unsuccessful conservative treatment; and confirmation of the DLSS through MRI or CT post-myelography in cases of MRI incompatibility. Exclusion criteria were: inability to complete the ODI, to walk less than 6 minutes or other neurologic disorders affecting gait. Moreover, we recruited healthy, age-matched subjects from the community surrounding the clinic. Inclusion criteria were: age $>40$; no history of back problems within the last 6 months before the study; no pain; no functional limitations. Exclusion criteria were neurological disorders affecting gait.

Surgical procedure: All patients received, depending on their clinical and imaging diagnostics, decompression surgery only or decompression combined with fusion surgery [15]. Fusion was achieved either by posterolateral fusion or by TLIF (transforaminal lumbar interbody fusion). For posterolateral fusion, patients received a pedicle screw-rod system with autologous bone apposition from either local bone or the iliac crest [16]. For TLIF, according to the standard of this technique after the discectomy additionally a cage filled with autologous bone was implanted from posterolateral into the intervertebral space [16]. For decompression, either open surgery or microsurgical technique was used, and both were performed through a midline approach. In the microsurgical technique, uni- or bilateral fenestration and flavectomy was performed whereas in the open surgery the decompression was done via an interspinous approach with interlaminar flavectomy.

Experimental procedure: Radiological data were collected as part of standard clinical routine. Baseline measurements were taken on the day before surgery in our clinic. First, the patients completed the ODI questionnaire. Then, gait analysis was performed with inertial sensors 
attached to the patients' pelvis and measuring specific gait parameters during a 6-minute walking test. The test was repeated at the 10-week and 12-month follow up.

ODI questionnaire: The ODI is a standardized questionnaire comprising ten self-administered parameters quantifying the impact of low back pain on daily life [17-20]. The items are: pain intensity; personal care; lifting; walking; sitting; standing; sleeping; sexual life; social life; travelling. The results are presented as scores from 0 (no impact) to 100 (bed bound and extremely limited).

Pelvic rigidity during walking: Participants were asked to complete a 6-minute walk test [21, 22] involving walking back and forth a well-lit hallway for 6 minutes. During these walking trials, pelvic acceleration was measured in three planes (sagittal, frontal and transverse) to assess pelvic rigidity during walking. The inertial sensor (RehaGait ${ }^{\circledR}$ system, Hasomed GmbH, Magdeburg, Germany, dimensions $60 \mathrm{~mm} \times 15 \mathrm{~mm} \times 35 \mathrm{~mm}$ ) contained a triaxial accelerometer, gyroscope and magnetometer and was placed on the pelvis overlying L5 using double sided tape and a Velcro strap. The sensor measured the amplitude of the axis deviation as well as the acceleration in all three planes at 400 samples per second. The system has been shown to be valid and reliable in human subjects $[23,24]$. Root mean square (RMS) and relative RMS of the acceleration data normalized to walking speed during the middle 10 seconds of the walking trial during minute 3 of the 6-minute walk test were calculated in each plane and for the total signal according to Sekine et al. [10] Smaller relative accelerations corresponded to greater pelvic rigidity. 
Clinical parameters: Age, body mass index (BMI), duration of symptoms, muscle atrophy and degree of stenosis were taken from the electronic case history and corresponding magnetic resonance (MR) images, respectively. Duration of symptoms were categorized as $<2$ years, 2 to 5 years, and $>5$ years. The MR images were analyzed for the degree of the stenosis and the muscle atrophy at the level of the stenosis which was addressed by surgery. Muscle atrophy was measured using the Goutallier classification [25]. Accordingly, the atrophy was classified in four grades estimating the fatty infiltration as a parameter for atrophy. The measurement was carried out by a radiologist at our clinic who was blinded to the results of the gait test. In addition, bilateral cross-sectional areas (CSA) of the erector spinae and psoas muscles were quantified from MRIs using dedicated software (Syngo Via Siemens Healthcare, Erlangen, Germany). Absolute CSA for each muscle and relative side-to-side differences (\%) were used for further analyses. The degree of stenosis was graded according to the classification by Schizas et al. [26]. Schizas et al. divided the stenosis in four grades ranging from A (clearly visible cerebrospinal fluid) to D (no epidural fat or cerebrospinal fluid visible). This classification was performed by three independent observers. Pelvic incidence, pelvic tilt and sacral slope were measured on sagittal plane standing radiographs [27].

Statistical analysis: The statistical analysis was performed using SPSS Version 21 (IBM Corporation, Armonk, NY). A stepwise linear regression model was used to determine if age, BMI, duration of symptoms, lumbar muscle atrophy, pelvic rigidity and stenosis grade predict outcome after decompression surgery (change in ODI). Differences in pelvic rigidity during walking between healthy persons and patients before and after decompression surgery were detected using a linear mixed model with time as within-subject factor and group (patient vs. 
healthy) as between-subject factor and age and BMI as covariates. Cross correlations and a linear regression model were used to determine if lumbar muscle atrophy and stenosis grade correlate with pelvic rigidity during walking. The statistical significance level was set a priori to 0.05 .

\section{Results}

\section{Clinical parameters}

We included 29 patients with symptomatic DLSS in this study (12 women, 17 men; mean \pm 1 standard deviation (range), age: $72.5 \pm 5.8$ (59 to 84 ) years; BMI: $28.0 \pm 4.7$ (18.8 to 35.9 ) $\left.\mathrm{kg} / \mathrm{m}^{2}\right)$. Nine patients were lost to follow-up because they did not want to return to the clinic for measurements, but their data was still used in the statistical analyses at baseline. Sixteen patients had experienced symptoms for up to 2 years, 6 patients for 2 to 5 years, and 6 patients for more than 5 years (Table 1). Three patients had a muscle atrophy grade 3 , twelve patients a grade 2 , ten patients a grade 1 and one patient had no muscle atrophy (Table 1). CSAs of the erector spinae and psoas muscles ranged from 11 to $36 \mathrm{~cm}^{2}$ and from 2 to $24 \mathrm{~cm}^{2}$, respectively. Ten patients had a maximum stenosis of grade 4 , eight patients had a grade of 3 , six patients a grade of 2 and three patients a grade of 1 (Table 1). Three had no data available for the classification of the muscle atrophy due to MRI incompatibility. Only two patients did not have any classification for the degree of stenosis because with one patient, a classification via CT-Scan was possible. Pelvic incidence ranged from 31 to $83^{\circ}$, pelvic tilt from 8 to $50^{\circ}$, and sacral slope from 19 to $64^{\circ}$. We also included 23 healthy persons ( 12 women, 11 men; age: $60.2 \pm 10.6$ (43 to 86 ) years; BMI: $24.1 \pm 3.5$ (15.8 to 34.4$\left.) \mathrm{kg} / \mathrm{m}^{2}\right)$ for the walking assessments. 
Although walking speed was lower in patients than in healthy persons (patients, pre-operatively: $1.09 \pm 0.34 \mathrm{~m} / \mathrm{s} ; 10$-week follow-up: $1.16 \pm 0.28 \mathrm{~m} / \mathrm{s} ; 12$-month follow-up: $1.13 \pm 0.27 \mathrm{~m} / \mathrm{s}$; healthy persons, $1.29 \pm 0.27 \mathrm{~m} / \mathrm{s}$ ), these differences did not reach statistical significance after correcting for multiple comparisons $(\mathrm{P}>0.021)$.

Do duration of symptoms, lumbar muscle atrophy, pelvic rigidity and stenosis grade predict outcome after decompression surgery (ODI)?

Duration of symptoms, lumbar muscle atrophy, pelvic rigidity and stenosis grade did not explain changes in ODI from before to after surgery. BMI was the only parameter included into the stepwise linear regression model for explaining changes in ODI from baseline to 10 -week follow-up (Table 2). Patients with greater BMI had a greater improvement in ODI from baseline to 10 -week follow-up. The variability in BMI explained $25.3 \%$ of variance in the change in ODI from baseline to 10-week follow-up. None of the included parameters explained the change in ODI from baseline to 12-month follow-up.

Does pelvic rigidity during walking differ between patients before and after decompression surgery and healthy persons?

Pelvic rigidity in the transverse plane during walking in patients before and 10-week and 12months after surgery was higher than in healthy persons reflected in lower relative pelvis accelerations in the medio-lateral and antero-posterior directions (Tables 3 and 4). Pelvic rigidity in the vertical direction during walking in patients before and 10 weeks and 12 months after surgery was lower than in healthy persons (Tables 3 and 4). Pelvic rigidity in the transverse plane increased in antero-posterior direction from baseline to 10 -week follow-up and decreased 
in antero-posterior and medio-lateral direction from 10-week to 12-week follow-up (Tables 3 and 4). Overall, pelvic rigidity in the patients converged towards pelvic rigidity in a healthy population 12 months postoperatively.

Do lumbar muscle atrophy and stenosis grade correlate with pelvic rigidity and pelvic tilt? Grade of stenosis correlated with pelvic rigidity in all directions (Table 5). Patients with greater stenosis grade had greater pelvic rigidity in the transverse plane reflected by smaller pelvis accelerations. Moreover, patients with greater medio-lateral pelvic rigidity also had greater antero-posterior but smaller vertical pelvic rigidity $\left(\mathrm{R}^{2}=0.310\right.$ and 0.537 ; both $\mathrm{P}<0.009$, respectively; Table 5). Lumbar muscle atrophy did not correlate with pelvic rigidity during walking. However, patients with lower stenosis grade had greater qualitative muscle atrophy $\left(\mathrm{R}^{2}=0.175, \mathrm{P}=0.040\right)$ and patients with smaller erector spinae and psoas muscle CSA had a greater pelvis tilt $\left(\mathrm{R}^{2}>0.344, \mathrm{P}<0.010\right.$, Figure 2$)$. Patients with greater body mass generally had greater erector spinae muscle CSA $(\mathrm{R}>0.476 ; \mathrm{P}<0.029)$ and taller patients had greater psoas muscle CSA $(\mathrm{R}>0.487 ; \mathrm{P}<0.025)$. BMI did not correlate with erector spinae or psoas muscle CSA $(\mathrm{R}<0.349 ; \mathrm{P}>0.123)$. The association between pelvic tilt and erector spinae and psoas muscle CSA were still significant after correcting muscle CSA for body mass or body height $\left(\mathrm{R}^{2}>0.222 ; \mathrm{P}<0.049\right)$

\section{Discussion}

The aim of this study was to determine the association among the duration of symptoms, lumbar muscle atrophy, grade of stenosis and pelvic rigidity during walking in patients with symptomatic DLSS treated with either decompression or decompression and fusion surgery and 
compared to a healthy control group. While a higher BMI predicted a greater improvement in clinical scores, duration of symptoms and lumbar muscle atrophy did not seem to play a role. In our study, patients walked with a more rigid pelvis in the transverse plane than healthy persons. Moreover, pelvic rigidity decreased after decompression surgery converging towards the pelvic rigidity of healthy people. Thus, our results suggest that pelvic movement in the transverse plane plays an important role in DLSS.

Our results showed that neither duration of symptoms, lumbar muscle atrophy nor stenosis grade correlate with changes in ODI after surgery. The only independent predictive factor in the linear regression model to explain changes in ODI was BMI. Patients with greater BMI had a greater improvement from baseline to 1-year follow-up. However, ODI at baseline was not associated with BMI at baseline. Hence, this observation was not explained by poorer ODI at baseline in patients with greater BMI. It is well known that, in general, obese patients have more postoperative complications such as wound infections [28]. In the literature, the data about BMI as a predictive factor remains inconclusive. While Elsayed et al. [28] support our findings, other studies show less favorable clinical outcome in obese patients [29, 30]. Overall, our findings are consistent with the current state of research regarding predictive factors for the outcome after decompression surgery in lumbar spinal stenosis [4, 6, 7, 31-33]. Further studies are needed to better understand the clinical significance of BMI in the postoperative outcome in patients with DLSS.

To date, little is known about the predictive value of the duration of symptoms for DLSS outcome. In a prospective study in 105 patients, Jönsson et al. [7] found that patients with longer 
ongoing symptoms did not show a worse post-operative clinical outcome. Similarly, we did not find an association between duration of symptoms and clinical outcome. While some studies have examined the role of paraspinal muscle degeneration in DLSS in conservative treatment or pre-operatively [31,34], little is known about the role of muscle degeneration in postoperative outcome [35]. In DLSS, the grade of muscle atrophy does not seem to correlate with the severity of symptoms [31, 34, 36]. Gellhorn et al. [31] reported in a longitudinal study involving 209 patients that the CSA of lumbar muscles did not correlate with the functional status of patients with symptomatic DLSS and was not a predictive parameter for pain and function at 6- and 12months follow-ups in conservative treatment. In contrast, two smaller studies reported contradicting results $[34,36]$. Fortin et al. [6] found in a retrospective, multi-center cohort involving 35 patients that greater multifidus fatty infiltration and lower psoas muscle relative CSA were associated with higher ODI and pain interference scores. However, these studies only highlighted the correlation between muscle atrophy and clinical findings but did not consider it as a predictive factor in mid- and long-term outcome after decompression surgery. In the only previous study reporting postoperative data, Zotti et al. [30] suggested a better clinical outcome in patients with less muscle atrophy. These authors observed a correlation between greater multifidus muscle atrophy relative to the CSA of the psoas muscle with less improvement in ODI after decompression surgery in a retrospective cohort including 66 patients. In contrast, in our study, we did not find a correlation between the degree of lumbar muscle atrophy and the pre- to postoperative change in ODI. However, we found that patients with smaller erector spinae and psoas muscle CSA had greater pelvis tilt. Dohzono et al. [35] reported that more severely affected patients had less pelvic tilt. While we also observed that patients with a higher degree of stenosis had less pelvic tilt, our data did not reach statistical significance $(\mathrm{R}=0.383, \mathrm{P}=0.071)$. 
Combining our results with the data of previous studies, one can summarize that preoperatively patients with DLSS seem to have less pelvic tilt and walk with a more rigid pelvic than healthy controls [31, 34-36]. This observation may reflect upon a compensating mechanism in posture and gait by reducing the lumbar lordosis through pelvic flexion with consequently lower pelvic tilt to open up the lumbar spinal canal. The associated greater pelvic rigidity may be needed to stabilize the dysbalanced lumbo-pelvic region because of the more flexed pelvis. It is possible that patients with more severe stenosis require greater muscle forces to stabilize the region of the stenosis and that this greater demand may act as training of these muscles. This could explain the stated difference of muscle atrophy, which was less severe in higher degrees of stenosis.

While preoperatively there is extensive evidence that stenosis grade does not correlate with clinical features $[4,7,32]$, postoperatively there is limited literature describing such association. In a prospective cohort study of 459 patients, Otani et al. did not find any correlation between degree of stenosis and clinical 1-year outcome after decompression surgery [37]. Our data support these findings, and hence informing patients on the limited predictability of the clinical outcome is warranted to not provoke unrealistic expectations in patients with radiographically severe stenosis.

To the best of our knowledge, this is the first study examining pelvic rigidity during walking in patients with DLSS. We observed a difference in pelvic accelerations in all three anatomic directions compared to healthy subjects except at the 12-month follow up in antero-posterior direction. Furthermore, pelvic rigidity decreased from baseline to 12-month follow-up in the antero-posterior direction and from the 10-week to the 12-month follow-up in the medio-lateral 
direction. Other studies have shown that patients with symptomatic DLSS have less lumbar lordosis, decreased hip extension und decreased stride length during walking compared to healthy persons [30]. Presumably, lumbar and hip flexion lead to an opening of the spinal canal and therefore lessen the clinical symptoms of DLSS. With the hip flexed, the pelvic tilt is smaller. To increase stride length, the hip needs to be extended thus narrowing the spinal canal. In order to keep the spinal canal more open, patients seem to maintain a protective posture with hips and lumbar spine in a flexed position. As a physiological, theoretical explanation of our results, patients with DLSS seem to maintain this posture with hip and lumbar spine in a continuous, stable flexed position as much as possible. This argument is further supported by our observation of a decrease in pelvic rigidity after decompression surgery. We hypothesize that due to the newly open spinal canal after decompression surgery it is no longer necessary to keep the spine in a stable, flexed position to prevent neurologic claudication hence resulting in lower postoperative pelvic rigidity during walking that converges towards the lower pelvic rigidity in healthy people. However, greater pelvic rigidity in the transverse plane during walking appears to come at the cost of greater vertical accelerations observed in our study presumably representing greater compressive forces. The implications of such greater compressive forces have yet to be elucidated. Overall, pelvic rigidity during walking might by a sensitive parameter for the early detection of adaption mechanisms due to DLSS. Whether these patients could benefit from specific rehab programs should be investigated in further studies.

Lumbar muscle atrophy did not correlate with pelvic rigidity during walking but patients with smaller erector spinae and psoas CSA had greater pelvic tilt. As shown in previous studies, greater muscular atrophy is present in DLSS compared to controls [38] but does not increase 
with greater degree of stenosis $[31,34,36]$. Interestingly, in our study we observed a negative correlation between muscular atrophy and severity of stenosis: patients with lower stenosis grade had greater muscle atrophy. This might be due to competing influences on paraspinal muscles in patients with DLSS. First, neurological claudication and pain lead to inactivity. Inactivity would lead to a decrease in the use of paraspinal muscle, thus leading to a decrease in CSA. Combined with segmental denervation in DLSS, this leads to a decrease of paraspinal muscles in volume and an increase in fatty infiltration. In advanced DLSS, maintaining a protective posture becomes more important. Hence, the paraspinal muscles are contracted during walking and standing, and keeping that posture may have a training effect on these muscles. However, due to segmental denervation it might be that a normal muscular mass can no longer be maintained. This would explain why, even though patients with DLSS have greater muscle atrophy than healthy people, the association between muscular atrophy and severity of the stenosis may not be positive but negative as observed in our study or not be detectable as reported by other studies $[31,34,36]$.

The main strengths of this study were objective measurements of pelvis rigidity during walking using an inertial sensor system. To evaluate the radiographic parameters, three independent observers were involved. Our patients were heterogeneous regarding duration of symptoms and degree of stenosis. Microsurgical or open decompression surgery without or with stabilization depending on the indication varies greatly in operation time and approach morbidity and therefore might influence the postoperative rehabilitation process. The cohort group was slightly older than the control group. Further, the predominantly elderly patients in this study frequently have comorbidities constituting possible bias. Many patients with symptomatic DLSS complete 
their treatment between 3 and 6 months after surgery. Additional follow-ups during the first 12 months after surgery may have revealed subject specific recovery patterns. Moreover, additional postoperative radiological assessments were not performed because these are not part of routine care. Thus, we do not have any information about the postoperative changes in muscular atrophy, muscle CSA or pelvic tilt. Nonetheless, the results clearly revealed preoperative muscular and posture deficits and changes in pelvic rigidity during walking that may be addressed by prehabilitation and rehabilitation approaches aimed at improving the outcome of decompression surgery in patients with symptomatic DLSS.

\section{Conclusion}

Duration of symptoms, lumbar muscle atrophy and stenosis did not predict the outcome after decompression surgery. The only independent factor correlating positively with a greater change in ODI postoperatively was a higher BMI. We did not reveal a correlation between muscle atrophy and pelvic rigidity during walking. Nevertheless, patients with lower stenosis grade had a greater muscle atrophy, and patients with smaller CSA had greater pelvic tilt. Patients with DLSS had greater pelvic rigidity in the transverse plane assessed as lower pelvic acceleration during walking in antero-posterior and medio-lateral direction compared to healthy people. Patients with greater stenosis grade had a higher pelvic rigidity, and pelvic rigidity decreased after decompression surgery converging towards the pelvic rigidity of healthy people. Pelvic rigidity during walking may be a useful screening parameter for identifying early compensating mechanisms. Whether it can be used as a parameter for personalized treatment planning or outcome prognosis necessitates further evaluation. 


\section{References}

1. Steurer J, Nydegger A, Held U, et al. LumbSten: the lumbar spinal stenosis outcome study. BMC Musculoskelet Disord. 2010;11:254. https://doi.org/10.1186/1471-2474-11-254.

2. Kalichman L, Cole R, Kim DH, et al. Spinal stenosis prevalence and association with symptoms: the Framingham Study. Spine J. 2009;9(7):545-50.

https://doi.org/10.1016/j.spinee.2009.03.005.

3. Zaina F, Tomkins-Lane C, Carragee E, Negrini S. Surgical Versus Nonsurgical

Treatment for Lumbar Spinal Stenosis. Spine (Phila Pa 1976). 2016;41(14):E857-68. https://doi.org/10.1097/BRS.0000000000001635.

4. Yarjanian JA, Fetzer A, Yamakawa KS, Tong HC, Smuck M, Haig A. Correlation of paraspinal atrophy and denervation in back pain and spinal stenosis relative to asymptomatic controls. PM R. 2013;5(1):39-44. https://doi.org/10.1016/j.pmrj.2012.08.017.

5. Chen YY, Pao JL, Liaw CK, Hsu WL, Yang RS. Image changes of paraspinal muscles and clinical correlations in patients with unilateral lumbar spinal stenosis. Eur Spine J.

2014;23(5):999-1006. https://doi.org/10.1007/s00586-013-3148-z.

6. Fortin M, Dobrescu O, Courtemanche M, et al. Association Between Paraspinal Muscle Morphology, Clinical Symptoms, and Functional Status in Patients With Degenerative Cervical Myelopathy. Spine (Phila Pa 1976). 2017;42(4):232-9. https://doi.org/10.1097/BRS.0000000000001704.

7. Jonsson B, Annertz M, Sjoberg C, Stromqvist B. A prospective and consecutive study of surgically treated lumbar spinal stenosis. Part I: Clinical features related to radiographic findings. Spine (Phila Pa 1976). 1997;22(24):2932-7. 
8. Igawa T, Katsuhira J, Hosaka A, Uchikoshi K, Ishihara S, Matsudaira K. Kinetic and kinematic variables affecting trunk flexion during level walking in patients with lumbar spinal stenosis. PLoS One. 2018;13(5):e0197228. https://doi.org/10.1371/journal.pone.0197228.

9. Kang MH, Kim ER, Kim YG, Kim TH, Oh JS. The effects of lumbo-pelvic postural taping on gait parameters in patients with lumbar spinal stenosis. Clin Biomech (Bristol, Avon). 2013;28(9-10):956-60. https://doi.org/10.1016/j.clinbiomech.2013.10.005.

10. Sekine M, Tamura T, Yoshida M, et al. A gait abnormality measure based on root mean square of trunk acceleration. J Neuroeng Rehabil. 2013;10:118. https://doi.org/10.1186/17430003-10-118.

11. Kuwahara W, Deie M, Fujita N, et al. Characteristics of thoracic and lumbar movements during gait in lumbar spinal stenosis patients before and after decompression surgery. Clin Biomech (Bristol, Avon). 2016;40:45-51. https://doi.org/10.1016/j.clinbiomech.2016.10.016.

12. Byrnes SK, Nuesch C, Loske S, et al. Inertial Sensor-Based Gait and Attractor Analysis as Clinical Measurement Tool: Functionality and Sensitivity in Healthy Subjects and Patients With Symptomatic Lumbar Spinal Stenosis. Front Physiol. 2018;9:1095. https://doi.org/10.3389/fphys.2018.01095.

13. Herndon CL, Horodyski M, Vincent HK. Acute effects of anesthetic lumbar spine injections on temporal spatial parameters of gait in individuals with chronic low back pain: A pilot study. Gait Posture. 2017;58:369-73. https://doi.org/10.1016/j.gaitpost.2017.08.016.

14. Loske S, Nuesch C, Byrnes KS, et al. Decompression surgery improves gait quality in patients with symptomatic lumbar spinal stenosis. Spine J. 2018;18(12):2195-204. https://doi.org/10.1016/j.spinee.2018.04.016. 
15. Fleege C, Rickert M, Rauschmann M. [The PLIF and TLIF techniques. Indication, technique, advantages, and disadvantages]. Orthopade. 2015;44(2):114-23. https://doi.org/10.1007/s00132-014-3065-9.

16. Conrad BP, Shokat MS, Abbasi AZ, Vincent HK, Seay A, Kennedy DJ. Associations of self-report measures with gait, range of motion and proprioception in patients with lumbar spinal stenosis. Gait Posture. 2013;38(4):987-92. https://doi.org/10.1016/j.gaitpost.2013.05.010.

17. Fairbank JC, Couper J, Davies JB, O'Brien JP. The Oswestry low back pain disability questionnaire. Physiotherapy. 1980;66(8):271-3.

18. Fairbank JC, Pynsent PB. The Oswestry Disability Index. Spine (Phila Pa 1976). 2000;25(22):2940-52; discussion 52.

19. Mannion AF, Junge A, Fairbank JC, Dvorak J, Grob D. Development of a German version of the Oswestry Disability Index. Part 1: cross-cultural adaptation, reliability, and validity. Eur Spine J. 2006;15(1):55-65. https://doi.org/10.1007/s00586-004-0815-0.

20. Mannion AF, Junge A, Grob D, Dvorak J, Fairbank JC. Development of a German version of the Oswestry Disability Index. Part 2: sensitivity to change after spinal surgery. Eur Spine J. 2006;15(1):66-73. https://doi.org/10.1007/s00586-004-0816-z.

21. Laboratories ATSCoPSfCPF. ATS statement: guidelines for the six-minute walk test. Am J Respir Crit Care Med. 2002;166(1):111-7. https://doi.org/10.1164/ajrccm.166.1.at1102.

22. Balke B. A Simple Field Test for the Assessment of Physical Fitness. Rep 63-6. Rep Civ Aeromed Res Inst US. 1963:1-8.

23. Donath L, Faude O, Lichtenstein E, Nuesch C, Mundermann A. Validity and reliability of a portable gait analysis system for measuring spatiotemporal gait characteristics: comparison to 
an instrumented treadmill. J Neuroeng Rehabil. 2016;13:6. https://doi.org/10.1186/s12984-016$\underline{0115-\mathrm{z} .}$

24. Donath L, Faude O, Lichtenstein E, Pagenstert G, Nuesch C, Mundermann A. Mobile inertial sensor based gait analysis: Validity and reliability of spatiotemporal gait characteristics in healthy seniors. Gait Posture. 2016;49:371-4. https://doi.org/10.1016/j.gaitpost.2016.07.269. 25. Goutallier D, Postel JM, Bernageau J, Lavau L, Voisin MC. Fatty muscle degeneration in cuff ruptures. Pre- and postoperative evaluation by CT scan. Clin Orthop Relat Res. 1994;(304):78-83.

26. Schizas C, Theumann N, Burn A, et al. Qualitative grading of severity of lumbar spinal stenosis based on the morphology of the dural sac on magnetic resonance images. Spine (Phila Pa 1976). 2010;35(21):1919-24. https://doi.org/10.1097/BRS.0b013e3181d359bd.

27. Roussouly P, Nnadi C. Sagittal plane deformity: an overview of interpretation and management. Eur Spine J. 2010;19(11):1824-36. https://doi.org/10.1007/s00586-010-1476-9.

28. Elsayed G, Davis MC, Dupepe EC, et al. Obese (Body Mass Index >30) Patients Have Greater Functional Improvement and Reach Equivalent Outcomes at 12 Months Following Decompression Surgery for Symptomatic Lumbar Stenosis. World Neurosurg. 2017;105:884-94. https://doi.org/10.1016/j.wneu.2017.06.072.

29. Burgstaller JM, Held U, Brunner F, et al. The Impact of Obesity on the Outcome of Decompression Surgery in Degenerative Lumbar Spinal Canal Stenosis: Analysis of the Lumbar Spinal Outcome Study (LSOS): A Swiss Prospective Multicenter Cohort Study. Spine (Phila Pa 1976). 2016;41(1):82-9. https://doi.org/10.1097/BRS.0000000000001128.

30. Zotti MGT, Boas FV, Clifton T, Piche M, Yoon WW, Freeman BJC. Does pre-operative magnetic resonance imaging of the lumbar multifidus muscle predict clinical outcomes following 
lumbar spinal decompression for symptomatic spinal stenosis? Eur Spine J. 2017;26(10):258997. https://doi.org/10.1007/s00586-017-4986-x.

31. Gellhorn AC, Suri P, Rundell SD, et al. Lumbar Muscle Cross-Sectional Areas Do Not Predict Clinical Outcomes in Adults With Spinal Stenosis: A Longitudinal Study. PM R. 2017;9(6):545-55. https://doi.org/10.1016/j.pmrj.2016.09.014.

32. Athiviraham A, Yen D, Scott C, Soboleski D. Clinical correlation of radiological spinal stenosis after standardization for vertebral body size. Clin Radiol. 2007;62(8):776-80. https://doi.org/10.1016/j.crad.2007.02.011.

33. Mannion AF, Fekete TF, Pacifico D, et al. Dural sac cross-sectional area and morphological grade show significant associations with patient-rated outcome of surgery for lumbar central spinal stenosis. Eur Spine J. 2017;26(10):2552-64. https://doi.org/10.1007/s00586-017-5280-7.

34. Lohman CM, Tallroth K, Kettunen JA, Lindgren KA. Comparison of radiologic signs and clinical symptoms of spinal stenosis. Spine (Phila Pa 1976). 2006;31(16):1834-40. https://doi.org/10.1097/01.brs.0000227370.65573.ac.

35. Dohzono S, Toyoda H, Matsumoto T, Suzuki A, Terai H, Nakamura H. The influence of preoperative spinal sagittal balance on clinical outcomes after microendoscopic laminotomy in patients with lumbar spinal canal stenosis. J Neurosurg Spine. 2015;23(1):49-54. https://doi.org/10.3171/2014.11.SPINE14452.

36. Fortin M, Lazary A, Varga PP, Battie MC. Association between paraspinal muscle morphology, clinical symptoms and functional status in patients with lumbar spinal stenosis. Eur Spine J. 2017;26(10):2543-51. https://doi.org/10.1007/s00586-017-5228-y. 
37. Otani K, Kikuchi SI, Nikaido T, Konno SI. Magnitude of dural tube compression does not show a predictive value for symptomatic lumbar spinal stenosis for 1-year follow-up: a prospective cohort study in the community. Clin Interv Aging. 2018;13:1739-46. https://doi.org/10.2147/CIA.S171049.

38. Jiang J, Wang H, Wang L, et al. Multifidus Degeneration, A New Risk Factor for Lumbar Spinal Stenosis: A Case-Control Study. World Neurosurg. 2017;99:226-31. https://doi.org/10.1016/j.wneu.2016.11.142. 


\section{Figure captions}

Figure 1. Example MRI stenosis grade [33] and lumbar muscle atrophy [25].

Figure 2. Association between pelvic tilt and cross-sectional area of the left psoas muscle. 
Table 1. Baseline demographic and clinical data of patients with symptomatic DLSS.

\begin{tabular}{|c|c|c|c|c|c|c|c|c|c|}
\hline$I D$ & $\operatorname{Sex}$ & $\begin{array}{c}\text { Age } \\
\text { (years) }\end{array}$ & $\begin{array}{c}B M I \\
\left(\mathrm{~kg} / \mathrm{m}^{2}\right)\end{array}$ & $\begin{array}{c}\text { Duration of } \\
\text { symptoms } \\
\text { (years) }\end{array}$ & $\begin{array}{l}\text { Lumbar } \\
\text { muscle } \\
\text { atrophy }\end{array}$ & $\begin{array}{l}\text { Stenosis } \\
\text { grade }\end{array}$ & $\begin{array}{c}\text { ODI pre- } \\
\text { OP }\end{array}$ & $\begin{array}{c}\text { ODI 10- } \\
\text { weeks } \\
\text { post-OP }\end{array}$ & $\begin{array}{c}\text { ODI 12- } \\
\text { months } \\
\text { post-OP }\end{array}$ \\
\hline 1 & $\mathrm{~m}$ & 83 & 24.3 & 5.0 & 2.0 & 3.0 & 10 & 4 & 4 \\
\hline 2 & $\mathrm{f}$ & 75 & 23.8 & 2.0 & 2.0 & 2.0 & 14 & 2 & n.a. \\
\hline 3 & $\mathrm{~m}$ & 65 & 18.8 & 10.0 & 1.0 & 4.0 & 50 & 4 & 4 \\
\hline 4 & $\mathrm{f}$ & 66 & 23.0 & 2.0 & 1.0 & 2.0 & 8 & 2 & 6 \\
\hline 5 & $\mathrm{~m}$ & 58 & 30.2 & 2.0 & 2.0 & 3.0 & 66 & 34 & n.a. \\
\hline 6 & $\mathrm{~m}$ & 68 & 25.5 & 10.0 & 2.0 & 3.0 & 28 & 2 & 0 \\
\hline 7 & $\mathrm{~m}$ & 67 & 33.1 & 10.0 & 2.0 & 2.0 & 42 & 22 & n.a. \\
\hline 8 & $\mathrm{f}$ & 62 & 35.7 & 5.0 & 1.0 & 4.0 & 34 & 51 & 60 \\
\hline 9 & $\mathrm{f}$ & 82 & 32.4 & 10.0 & 2.0 & 4.0 & 22 & 22 & 13 \\
\hline 10 & $\mathrm{~m}$ & 74 & 24.8 & 2.0 & n.a. & 4.0 & 26 & 33 & 42 \\
\hline 11 & $\mathrm{f}$ & 78 & 30.1 & 5.0 & 3.0 & 1.0 & 0 & 0 & 0 \\
\hline 12 & $\mathrm{f}$ & 74 & 27.7 & 5.0 & 2.0 & 1.0 & 54 & 48 & 50 \\
\hline 13 & $\mathrm{~m}$ & 73 & 30.8 & 2.0 & 2.0 & 3.0 & 4 & 4 & 6 \\
\hline 14 & $\mathrm{~m}$ & 70 & 28.4 & 2.0 & 2.0 & 2.0 & 54 & 7 & n.a. \\
\hline 15 & $\mathrm{~m}$ & 76 & 22.7 & 2.0 & n.a. & n.a. & 16 & 0 & n.a. \\
\hline 16 & $\mathrm{f}$ & 71 & 33.3 & 2.0 & 2.0 & 3.0 & 28 & 12 & 26 \\
\hline 17 & $\mathrm{f}$ & 71 & 22.0 & 2.0 & 2.0 & 3.0 & 42 & 0 & 9 \\
\hline 18 & $\mathrm{~m}$ & 71 & 25.2 & 2.0 & 1.0 & 4.0 & 42 & 34 & 10 \\
\hline 19 & $\mathrm{f}$ & 78 & 35.4 & 2.0 & 1.0 & 4.0 & 50 & 42 & n.a. \\
\hline 20 & $\mathrm{~m}$ & 65 & 35.2 & 10.0 & 1.0 & 4.0 & 34 & 13 & n.a. \\
\hline 21 & $\mathrm{~m}$ & 79 & 24.5 & 5.0 & 1.0 & 2.0 & 42 & 4 & 22 \\
\hline 22 & $\mathrm{~m}$ & 79 & 26.3 & 2.0 & 3.0 & 4.0 & 47 & 5 & 36 \\
\hline 23 & $\mathrm{~m}$ & 72 & 25.0 & 5.0 & 1.0 & 4.0 & 22 & 0 & n.a. \\
\hline 24 & $\mathrm{f}$ & 70 & 24.8 & 2.0 & 1.0 & 4.0 & 29 & 2 & n.a. \\
\hline 25 & $\mathrm{~m}$ & 70 & 28.1 & 2.0 & 2.0 & 1.0 & 22 & 8 & 0 \\
\hline 26 & $\mathrm{f}$ & 68 & 27.6 & 5.0 & n.a. & n.a. & 28 & 0 & 0 \\
\hline 27 & $\mathrm{f}$ & 75 & 35.1 & 10.0 & 3.0 & 2.0 & 31 & 13 & 14 \\
\hline 28 & $\mathrm{~m}$ & 71 & 26.4 & 2.0 & 1.0 & 3.0 & 29 & 3 & 2 \\
\hline 29 & $\mathrm{~m}$ & 75 & 29.1 & 2.0 & 0.0 & 3.0 & 10 & 2 & 7 \\
\hline
\end{tabular}

$\mathrm{m}$-male; $\mathrm{f}-$ female; n.a.—not available; BMI—body mass index; ODI—Oswestry disability index. 
Table 2: Stepwise linear regression models explaining changes in the ODI from baseline to 10-week follow-up.

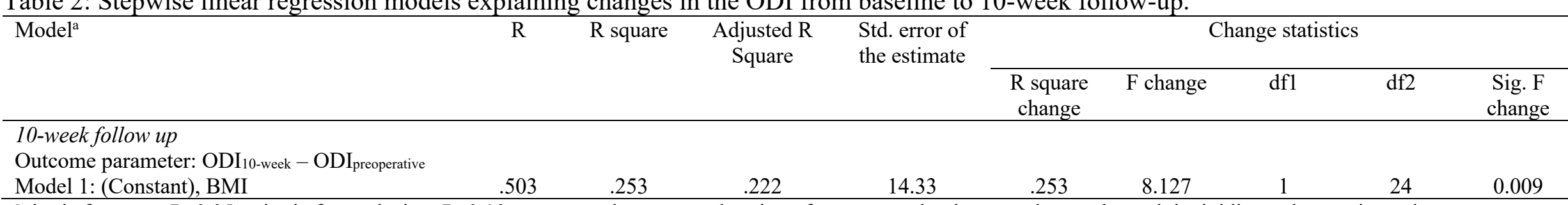

${ }^{a}$ Criteria for enter: $\mathrm{P}=0.05$; criteria for exclusion: $\mathrm{P}=0.10$; not entered were age, duration of symptoms, lumbar muscle atrophy, pelvis rigidity and stenosis grade. 
Table 3: Parameters describing pelvis stability at baseline and at 10-week and 12-months followup

\begin{tabular}{lcccc}
\hline \multirow{2}{*}{$\begin{array}{c}\text { Mean } \pm S D \\
\text { (min; max })\end{array}$} & $\begin{array}{c}\text { Patients with symptomatic LSS } \\
\text { Preoperative } \\
(N=29)\end{array}$ & $\begin{array}{c}\text { 10-week } \\
\text { postoperative } \\
(N=29)\end{array}$ & $\begin{array}{c}\text { 12-month } \\
\text { postoperative } \\
(N=20)\end{array}$ & $\begin{array}{c}\text { Healthy control } \\
\text { subjects } \\
(N=27)\end{array}$ \\
\hline relRMS & $0.950 \pm 0.034^{\mathrm{a}}$ & $0.959 \pm 0.024^{\mathrm{a}, \mathrm{b}}$ & $0.938 \pm 0.034^{\mathrm{a}}$ & $0.918 \pm 0.042$ \\
& $(0.800 ; 0.968)$ & $(0.897 ; 0.991)$ & $(0.834 ; 0.988)$ & $(0.814 ; 0.978)$ \\
relRMS & $0.247 \pm 0.108$ & $0.222 \pm 0.080^{\mathrm{b}}$ & $0.283 \pm 0.101$ & $0.302 \pm 0.102$ \\
& $(0.093 ; 0.594)$ & $(0.107 ; 0.386)$ & $(0.121 ; 0.536)$ & $(0.162 ; 0.563)$ \\
relRMS & $0.132 \pm 0.050^{\mathrm{b}}$ & $0.151 \pm 0.039^{\mathrm{b}}$ & $0.162 \pm 0.049$ & $0.180 \pm 0.039$ \\
& $(0.072 ; 0.221)$ & $(0.082 ; 0.222)$ & $(0.083 ; 0.265)$ & $(0.120 ; 0.280)$ \\
\hline
\end{tabular}

SD — standard deviation; relRMS - relative root mean square of the acceleration signal in V-vertical direction, APanteroposterior direction and ML-mediolateral direction according to Sekine et al. [10]; a - significant difference between times points in patients (ANOVA for repeated measures, $\mathrm{P}<0.05$ ); $\mathrm{b}$ - significantly different from healthy (t-test for independent samples, $\mathrm{P}<0.01$ ). 
Table 4: Differences in parameters describing pelvis stability between assessments points for patients and between patients and control subjects at each assessment.

\begin{tabular}{|c|c|c|c|c|c|c|}
\hline \multirow[b]{2}{*}{$\begin{array}{l}95 \% \text { confidence } \\
\text { intervals }\end{array}$} & \multicolumn{3}{|c|}{ Within patients } & \multicolumn{3}{|c|}{ Patients vs. control subjects } \\
\hline & $\begin{array}{c}\text { 10-week } \\
\text { postoperative vs. } \\
\text { preoperative }\end{array}$ & $\begin{array}{c}\text { 12-month } \\
\text { postoperative vs. } \\
\text { preoperative }\end{array}$ & $\begin{array}{c}\text { 12-week vs. 10- } \\
\text { week } \\
\text { postoperative }\end{array}$ & Preoperative & $\begin{array}{c}\text { 10-week } \\
\text { postoperative }\end{array}$ & $\begin{array}{c}\text { 12-month } \\
\text { postoperative }\end{array}$ \\
\hline relRMSV & {$[-0.004 ; 0.014]$} & {$[-0.010 ; 0.001]$} & {$[-0.019 ;-0.001]$} & {$[0.002 ; 0.043]$} & {$[0.011 ; 0.049]$} & {$[0.016 ; 0.034]$} \\
\hline relRMS $_{\mathrm{AP}}$ & {$[-0.031 ;-0.008]$} & {$[0.002 ; 0.032]$} & {$[0.001 ; 0.055]$} & {$[-0.115 ;-0.003]$} & {$[-0.131 ;-0.030]$} & {$[-0.0828 ; 0.044]$} \\
\hline relRMS & {$[-0.008 ; 0.010]$} & {$[0.003 ; 0.015]$} & {$[0.001 ; 0.016]$} & {$[-0.054 ;-0.008]$} & {$[-0.051 ;-0.007]$} & {$[-0.045 ;-0.010]$} \\
\hline
\end{tabular}


1 Table 5. Cross-correlations between muscle atrophy, stenosis grade and pelvic rigidity

2 parameters.

\begin{tabular}{|c|c|c|c|c|}
\hline $\begin{array}{l}\text { Pearson's correlation } \\
\text { coefficient (P-value) }\end{array}$ & $\begin{array}{c}\text { Lumbar muscle } \\
\text { atrophy }\end{array}$ & Stenosis grade & relRMS & relRMS $S_{A P}$ \\
\hline Stenosis grade & $\begin{array}{l}-0.418 \\
(0.034)\end{array}$ & & & \\
\hline $\operatorname{relRMS} S_{V}$ & $\begin{array}{l}-0.169 \\
(0.408)\end{array}$ & $\begin{array}{c}0.442 \\
(0.001)\end{array}$ & & \\
\hline $\operatorname{relRMS} S_{A P}$ & $\begin{array}{l}-0.118 \\
(0.565)\end{array}$ & $\begin{array}{l}-0.365 \\
(0.009)\end{array}$ & $\begin{array}{l}-0.120 \\
(0.534)\end{array}$ & \\
\hline $\operatorname{relRMS} S_{M L}$ & $\begin{array}{l}-0.039 \\
(0.849)\end{array}$ & $\begin{array}{r}-0.376 \\
(0.007)\end{array}$ & $\begin{array}{c}-0.733 \\
(<0.001)\end{array}$ & $\begin{array}{c}0.557 \\
(<0.001)\end{array}$ \\
\hline
\end{tabular}

3 relRMS - relative root mean square of the acceleration signal in V-vertical direction, AP-anteroposterior direction

4 and ML-mediolateral direction according to Sekine et al. [10].

5 


\begin{tabular}{l|l}
\hline \multicolumn{1}{c|}{ Stenosis grade classification by Schizas } & Muscle atrophy classification by Goutallier \\
\hline \multicolumn{1}{c}{ Grade A } \\
- Dural sac party occupied \\
by the rootlets \\
- Cerebrospinal fluid \\
clearly visible \\
- No stenosis
\end{tabular}




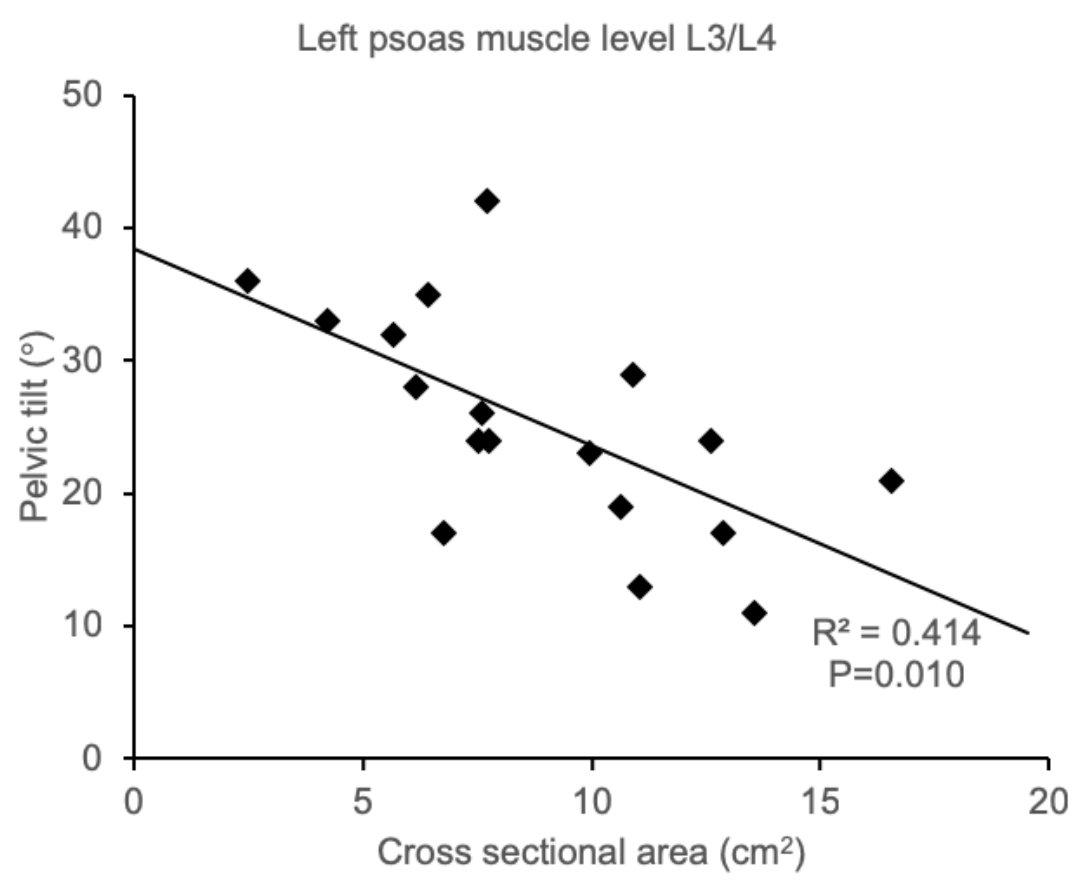

1 